\title{
A retrospective case series of segmental zoster paresis of limbs: clinical, electrophysiological and imaging characteristics
}

Ying Liu', Bing-Yun $\mathrm{Wu}^{1}$, Zhen-Shen $\mathrm{Ma}^{2}$, Juan-Juan Xu' ${ }^{1}$ Bing Yang ${ }^{3}$, Heng Li ${ }^{3}$ and Rui-Sheng Duan ${ }^{3 *}$

\begin{abstract}
Background: Segmental zoster paresis (SZP) of limbs, characterized by focal weakness of extremity, is recognized as a rare complication of herpes zoster $(\mathrm{HZ})$. The following study analyzes the clinical characteristics and data from electromyography and MRI scans in patients with motor weakness after zoster infection.

Methods: One thousand three hundred ninety-three patients from our database (Shandong Provincial Qianfoshan Hospital) suffering from $\mathrm{HZ}$ were retrospectively reviewed from June 2015 to July 2017. Patients who fulfilled the diagnostic criteria for SZP were included in the analysis. The clinical characteristics, as well as electromyography findings and MRI scans were analyzed.

Results: SZP was present in $0.57 \%$ of patients with $\mathrm{HZ}$ (8/1393). The average age of symptom onset in 8 SZP patients was 69 years old (SD: 13, range 47-87). The severity of muscle weakness ranged from mild to severe. The electrophysiological testing revealed the characteristics of axonopathy. Radiculopathy (2/8), plexopathy (2/8), radiculoplexopathy (3/8) and combined radiculopathy and mononeuropathy (1/8) were also identified. MRI revealed hyperintensity of the affected spinal dorsal horns, nerve roots or peripheral nerves.
\end{abstract}

Conclusions: SZP is associated with obvious limb weakness, nerve axons lesions and localization to nerve roots, plexus or peripheral nerves.

Keywords: Herpes zoster, Segmental zoster paresis, Infectious neuropathy, Nerve conduction, Electromyography, Nerve MRI

\section{Background}

Herpes zoster (HZ) is caused by varicella-zoster virus (VZV) which is latent in the dorsal root ganglia and reactivates when the immune system is not functioning properly. The incidence of $\mathrm{HZ}$ is about 4-4.5 per 1000 person-years [1], which is characterized by vesicular rash and burning pain. Postherpetic neuralgia is the most common neurologic syndrome of HZ, whereas segmental zoster paresis (SZP) of limbs is a relatively rare complication, characterized by focal weakness of upper or

\footnotetext{
* Correspondence: ruisheng_duan@yahoo.com

${ }^{3}$ Department of Neurology, Shandong Provincial Qianfoshan Hospital,

Shandong University, Jinan 250014, People's Republic of China

Full list of author information is available at the end of the article
}

lower extremity. The motor involvement can be observed in $0.5-5 \%$ patients with $\mathrm{HZ}[2,3]$.

The exact mechanism of SZP is not clear, although the spread of virus along the nerve is presumed [4-6]. The existing literature mainly includes case reports [7-18]. Comparatively, only few studies have described case series of SZP based on electrophysiological, imaging characteristics and prognosis data. In addition, antiviral medications and corticosteroids are the most commonly applied drugs for treating SZP. The prognosis for patients with SZP is generally favorable; however, in some extreme cases it can lead to permanent disability [2].

Here, we investigated clinical, electrophysiological and imaging evidence in a case series of 8 patients with SZP, as well as possible factors influencing prognosis.

(c) The Author(s). 2018 Open Access This article is distributed under the terms of the Creative Commons Attribution 4.0 International License (http://creativecommons.org/licenses/by/4.0/), which permits unrestricted use, distribution, and 


\section{Methods}

\section{Patients}

The patient database in Shandong Provincial Qianfoshan Hospital was reviewed for coded diagnoses of $\mathrm{HZ}$ from June 2015 to July 2017. Patients with HZ were diagnosed as SZP based on the following criteria [4]: 1) Infection of $\mathrm{HZ}$ preceding or following limb paresis established based on historical or physical examination evidence of a cutaneous vesicular eruption; 2) geographical (same limb) and temporal (no more than 30 days) evidence of associated limb weakness which was confirmed by a neurologist; 3) the lesions of nerve roots and plexus or mononeuropathy associated with $\mathrm{HZ}$ which were verified using electrophysiological testing.

Weakness was identified in each affected muscle as mild (corresponding to MRC 4 or $4+$ ), moderate (corresponding to MRC 3), severe (corresponding to MRC 2 or 1 ) and complete (corresponding to MRC 0 ).

According to the Medical Research Council scale, the muscle recovery was based on the following criteria: 1) complete recovery, if muscle strength was evaluated as grade 5 and the sensory symptom disappeared in the last follow-up visit; 2) no recovery, if no improvement in muscle strength was observed; 3) partial recovery, case between the complete and no recovery. In addition, all patients were followed up for approx. 0.5-2.0 years; the follow-up evaluation was based on sensorimotor symptom and muscle strength.

This retrospective study was approved by the medical ethics committee at our hospital.

\section{Electrophysiology}

Nihon Kohden MEB-9400 electromyograph was used to evaluate nerve injury. Surface electrodes were used to perform nerve conduction studies (NCS), including motor and sensory nerve conduction velocity (NCV), distal motor latency (DML), amplitudes of compound muscle action potentials $\left(\mathrm{CMAP}_{\mathrm{S}}\right)$ and sensory nerve action potentials $\left(\mathrm{SNAP}_{\mathrm{S}}\right)$.

Median motor study was performed with distal stimulation site over the median nerve at the wrist and proximal stimulation site at the antecubital fossa, recording the abductor pollicis brevis muscle. Ulnar motor study was performed with distal stimulation site over the ulnar nerve at the wrist and proximal stimulation site above the elbow, recording the abductor digiti minimi muscle. Radial motor study was performed with distal stimulation site in the forearm and proximal stimulation site in the arm, below the spiral groove, recording the extensor indicis proprius muscle.

Tibial motor study was performed with distal stimulation site slightly proximal and posterior to the medial malleolus and proximal stimulation site in the middle of the popliteal fossa, recording the abductor hallucis brevis muscle. Peroneal motor study was performed with distal stimulation site over the anterior ankle and proximal stimulation site below the fibular head, recording the extensor digitorum brevis muscle.

Axillary, suprascapular, musculocutaneous motor conduction studies were performed, recording the deltoid, supraspinatus and biceps brachii with surface electrodes repectively, and stimulation site was at Erb's point.

Antidromic and orthodromic sensory studies were performed in upper extremity or lower extremity respectively. Index finger and little finger were respectively stimulated to record SNAPs of the median and ulnar nerve.

A comparison with contralateral (asymptomatic) sides or normal values helped to determine the degree of damage. If $\mathrm{NCV}$ was slower than $75 \%$ of the lower limit of normal, and DML was longer than $130 \%$ of the upper limit of normal, it was regarded as demyelination. If the side-to-side amplitudes difference was greater than $50 \%$, it was regarded as abnormal and axonal lesion.

The needle electromyography (EMG) was performed by a concentric needle electrode, which was used to find spontaneous potentials such as positive sharp waves and fibrillation potentials conventionally graded from 0 to $4+$ as follows: 0 for none present; +1 for persistent single trains of potentials $(>2-3 \mathrm{~s})$ in at least two areas; +2 for moderate number of potentials in three or more areas; +3 for many potentials in all areas; +4 for full interference pattern of potentials. The firing pattern (activation, recruitment and interference pattern) was assessed.

According to SNAP and spontaneous activities in paraspinal muscles, associated limb was characterized as preganglionic, postganglionic lesions or combined preand postganglionic lesions, and then further identified as radiculopathy, plexopathy, radiculoplexopathy and peripheral nerves [4] .

\section{Imaging}

The MRI results were reviewed by an experienced radiologist. All studies included both T1- and T2- weighted images in coronal planes, without gadolinium-enhanced T1-weighted images. All T2-weighted sequences had fat saturation or a short tau inversion recovery sequence. Nerve root, plexus, or peripheral nerve images were identified as abnormal if prolonged nerve $\mathrm{T} 2$ or nerve enlargement was shown based on comparison with contralateral neural structures within the imaging field.

\section{Results}

\section{Patient demographics and clinical characteristics}

Eight out of 1393 inpatients with HZ fulfilled the diagnostic criteria for SZP, accounting for $0.57 \%$, which was confirmed by neurologic examination and electrodiagnostic evaluation. Clinical characteristics are listed in Table 1. 
Table 1 Demographics and clinical characteristics in patients with segmental zoster paresis

\begin{tabular}{ll}
\hline Features & \\
\hline Mean age (years) & 69 (range 47-87) \\
Men & 3 of 8 \\
Affected myotomes & \\
$\quad$ Left upper limb & 1 of 8 \\
Right upper limb & 5 of 8 \\
$\quad$ Left lower limb & 0 of 8 \\
Right lower limb & 2 of 8 \\
Rash after weakness (within 30 days) & 0 of 8 \\
Mean interval between rash and weakness (days) & 11.9 \\
Diabetes mellitus & 4 of 8 \\
Immunocompromise & 1 of 8 \\
Post-herpetic neuralgia 4 months after onset & 5 of 8 \\
Myotomes corresponding to dermatomes & 8 of 8 \\
Disseminated zoster & 1 of 8 \\
\hline
\end{tabular}

Detailed information of 8 patients are described in Table 2. Three descriptive cases are described in detail in the following paragraphs.

Rash distribution in all patients corresponded to weakness distribution (Fig. 1). Although the severity of muscle weakness ranged from mild to severe, most were at least moderate. All patients had paresthesia, such as tingling and numbness, corresponding to the distribution of affected myotomes. The deep tendon reflexes were diminished or absent in distribution of affected limbs. In addition, most patients had a certain degree of post-herpetic neuralgia 4 months after onset (5/8).

Four patients had diabetes (case 2, 3, 4 and 5), while 1 patient had a 20-year history of rheumatoid arthritis and use of immunosuppressive medications (case 5). Antiviral drugs, such as acyclovir, were administered to 5 patients (case 1, 2, 5, 6, 7) orally or intravenously within $72 \mathrm{~h}$ after the rash appeared, 3 patients after $72 \mathrm{~h}$. In addition, 4 patients received short term use of oral corticosteroids (case $2,3,5,8$ ); nevertheless, no patient experienced immediate pain or weakness relief after treatment.

The prognosis was quite different (Fig. 2). Patient 1 recovered completely 3 months after symptoms onset; this patient was placed on oral antiviral medication for 1 week. Patient 6 and 8 recovered partially through 1 or 0.5 year respectively. However, the remaining 5 patients did not recover until $0.5-2.0$ years of follow-up. Their muscle strength showed no improvements and one patient's muscles seemed atrophied (case 7).

\section{Electrophysiological characteristics}

All electrophysiological examinations were performed no more than 2 weeks after weakness onset. Briefly, nerve conduction studies showed markedly decreased or absent $\mathrm{CMAP}_{\mathrm{S}}$ and $\mathrm{SNAP}_{\mathrm{S}}$ amplitudes of affected nerves, compared with the contralateral sides (Table 3). Nerve conduction velocities of affected nerves were normal or mildly decreased, which means they were faster than $75 \%$ of the lower limit of normal and above $35 \mathrm{~m} / \mathrm{s}$. Distal motor latencies were shorter than $130 \%$ of the upper limit of normal. All of these showed axonal lesions, not demyelinating lesions (Fig. 3).

All the patients showed different grades of spontaneous potentials in affected muscles. The average grade was $2+$. There was a pattern of decreased recruitment of motor unit action potentials (MUAPs) in weak muscles. The positive sharp waves and fibrillation potentials in affected myotomes and the decreased recruitment of MUAPs suggested ongoing axonal lesions.

According to spontaneous activities in paraspinal muscles and $\mathrm{SNAP}_{\mathrm{S}}$ amplitudes, 2 among 8 were diagnosed with preganglionic lesions (case 1: C5-C6 radiculopathy; case 8: C5 radiculopathy), 2/8 were characterized as having postganglionic locations (case 2: upper and middle trunk lesions; case 4: lower trunk lesions) and 4 out of 8 were recognized as combined pre- and postganglionic lesions (case 3: L5-S1 radiculoplexopathy; case 5: C6-C8 radiculoplexopathy; case 6: L5 radiculoplexopathy; case 7: C7 radiculopathy and median, radial nerve lesions).

\section{Imaging characteristics}

The MRI examination was performed in 4 out of 8 patients no more than 1 week after electrophysiological testing (Fig. 4). Scans of affected brachial plexus nerves and corresponding spine segments, showed nerve enlargement and enhanced T2 signal intensity, and were in accordance with symptoms (case 5, 7 and 8). In patient 7 , median and radial nerve enlargement and T2 hyperintensity were observed, although abnormality of nerve root was not identified. One patient underwent an MRI scanning on cervical spine, not the affected nerves (case 2 ), and axial T2-weighted image showed hyperintensity in the dorsal horns of C5-6 spinal cord levels, corresponding to her sensory symptoms.

\section{Descriptive cases \\ Case 1}

A 47-year-old man developed severe burning pain and a vesicular eruption in the right shoulder and anterolateral arm. Two days later after the rash, he was not able to elevate his right arm to the shoulder level or bend the forearm at the elbow joint. Additionally, he also presented with numbness in the back of the thumb. Muscle weakness was present in the right deltoid (1/5), infraspinatus $(1 / 5)$, supraspinatus $(1 / 5)$ and biceps $(2 / 5)$, according to the MRC scale. Distal muscle strength was normal. The right biceps reflex was absent. The electrophysiological 


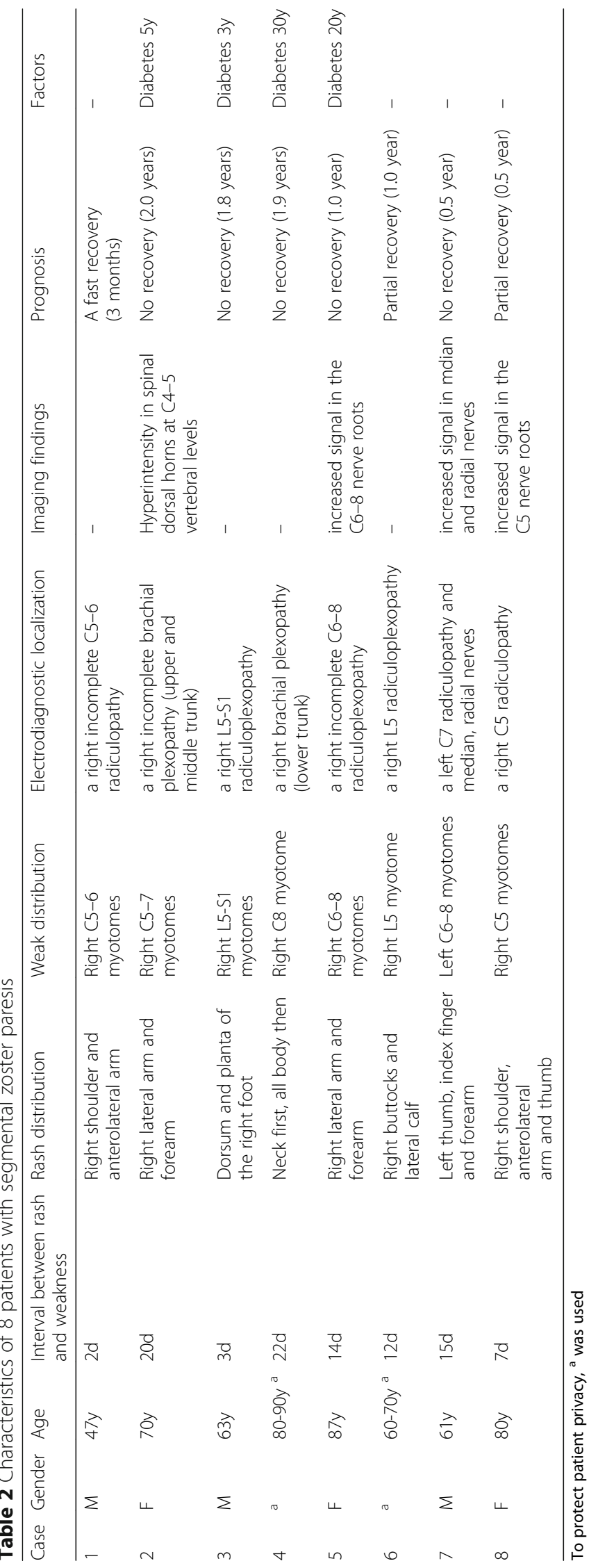




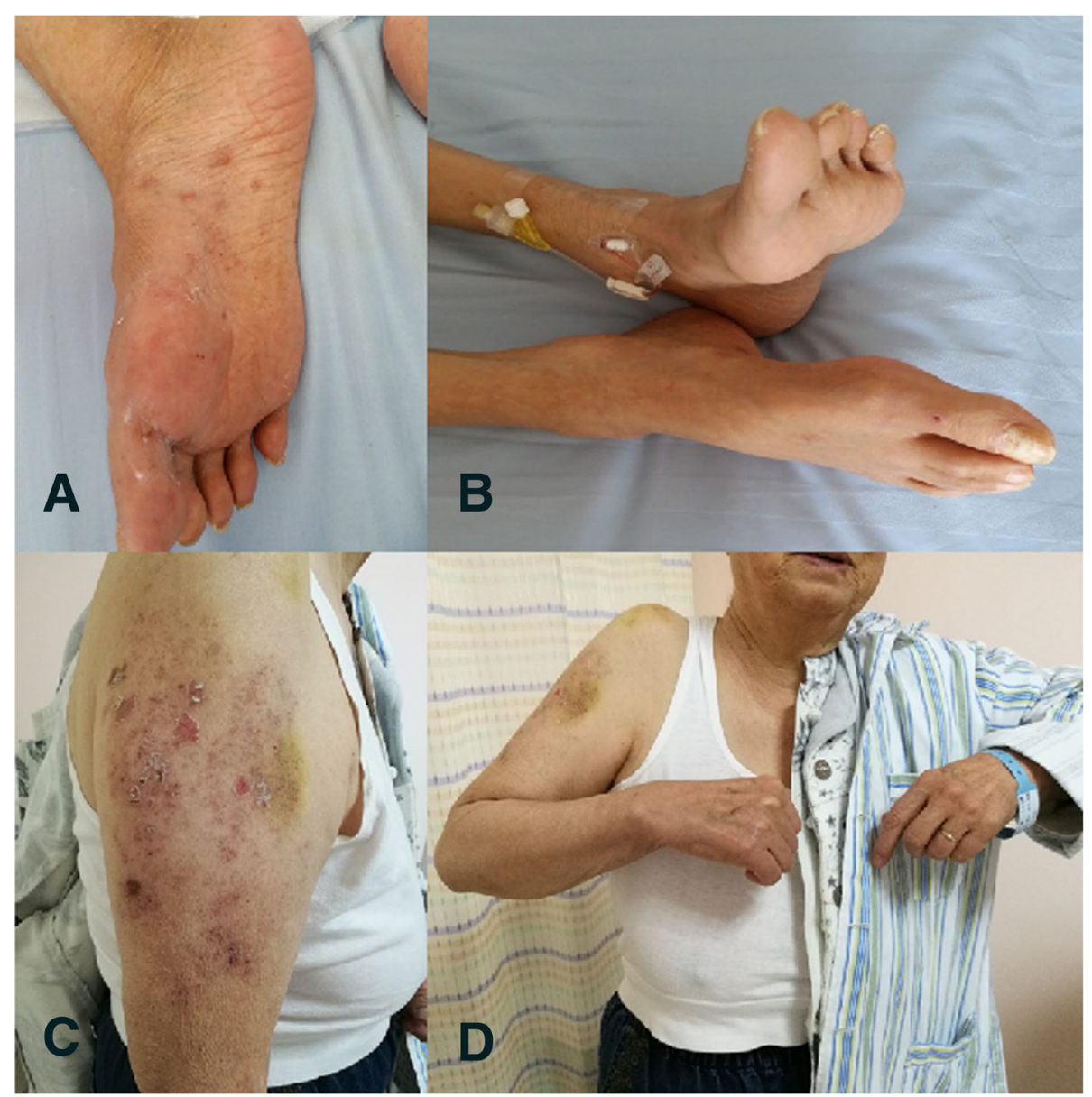

Fig. 1 Rash distribution in all patents corresponds to weakness distribution. Scars of a prior herpetic eruption and pigmentation over the dorsum and planta of the right foot were seen in patient 3, who had a foot drop (a and $\mathbf{b}$ ). Scars from prior herpetic eruption and pigmentation over the right shoulder and anterolateral arm were seen in patient 8, who could not elevate her shoulder (c and $\mathbf{d}$ )

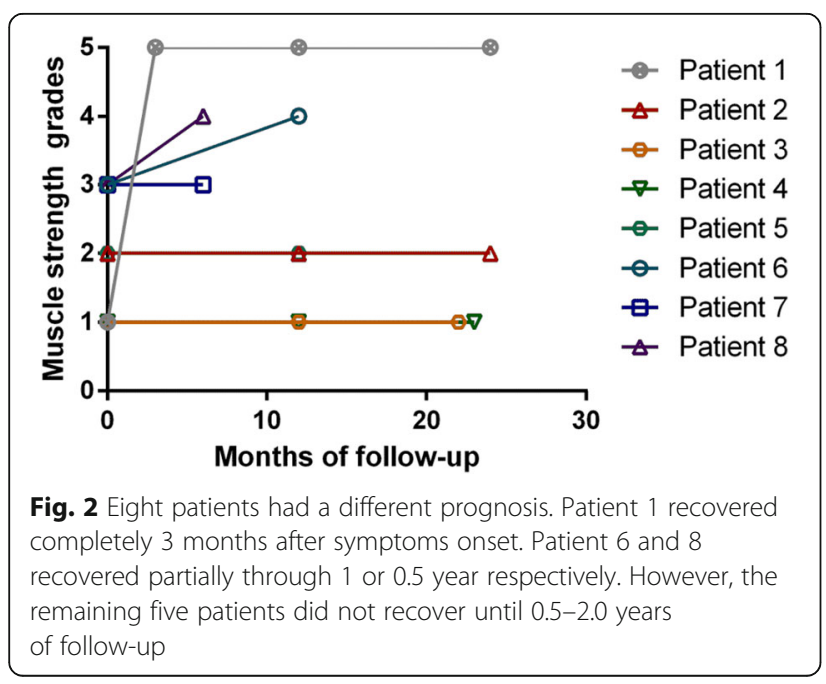

examination revealed lower amplitude axillary and musculocutaneous CMAPs (12.1 and $7.3 \mathrm{mV}$, respectively) compared to contralateral sides $(25.7$ and $18.9 \mathrm{mV}$, respectively). The sensory nerve conduction studies were normal. Abnormal spontaneous potentials and decreased recruitments of MUAPs were present in the right deltoid, infraspinatus, biceps and C5-6 paraspinal muscles. In conclusion, the electrophysiologic findings were consistent with the incomplete lesions of C5 and C6 nerve roots.

Within 3 months follow-up period, he regained the full arm strength without any treatments. Electrophysiologically, the amplitude axillary and musculocutaneous CMAPs (21.1 and $16.6 \mathrm{mV}$, respectively) were normal. Abnormal spontaneous activities in muscles innervated by $\mathrm{C} 5$ and $\mathrm{C} 6$ nerve roots disappeared and many polyphasic MUAPs were observed.

\section{Case 5}

An 87-year-old woman developed burning pain and vesicular rash over the right lateral arm and forearm. Two 
Table 3 Nerve conduction studies of patients with segmental zoster paresis

\begin{tabular}{|c|c|c|c|c|c|c|c|c|}
\hline & P1 & P2 & P3 & P4 & P5 & P6 & P7 & P8 \\
\hline \multicolumn{9}{|l|}{ CMAP of nerves $(\mathrm{mV})$} \\
\hline Axillary & $12.1(53 \% \downarrow)$ & $3.5(77 \% \downarrow)$ & & normal & $4.4(51 \% \downarrow)$ & & normal & $6.4(66 \% \downarrow)$ \\
\hline Suprascapular & & $1.7(84 \% \downarrow)$ & & & & & & $3.3(76 \% \downarrow)$ \\
\hline Musculocutaneous & $7.3(61 \% \downarrow)$ & $4.5(52 \% \downarrow)$ & & normal & $3.6(62 \% \downarrow)$ & & normal & normal \\
\hline Median & normal & normal & & $6.2(51 \% \downarrow)$ & $1.4(83 \% \downarrow)$ & & normal & normal \\
\hline Ulnar & normal & normal & & $5.3(53 \% \downarrow)$ & normal & & normal & normal \\
\hline Radial & normal & normal & & $5.2(55 \% \downarrow)$ & normal & & $4.1(63 \% \downarrow)$ & normal \\
\hline Peroneal & & & NR & & & $0.7(75 \% \downarrow)$ & & \\
\hline Tibial & & & $3.0(79 \% \downarrow)$ & & & normal & & \\
\hline \multicolumn{9}{|l|}{ SNAP of nerves $(\mu \mathrm{V})$} \\
\hline Median & normal & NR & & normal & NR & & $2.3(77 \% \downarrow)$ & normal \\
\hline Ulnar & normal & normal & & $2.8(60 \% \downarrow)$ & normal & & normal & normal \\
\hline Radial & normal & normal & & normal & NR & & $4.5(64 \% \downarrow)$ & normal \\
\hline Superficial peroneal & & & NR & & & NR & & \\
\hline Sural & & & NR & & & normal & & \\
\hline
\end{tabular}

The amplitudes of CMAPs and SNAPs were compared with contralateral side

CMAP compound muscle action potential, SNAP sensory nerve action potential, $\mu V$ microvolt, $m V$ millivolt, $N R$ no response

weeks after rash, she was not able to elevate her right arm to the shoulder level, bend the forearm at the elbow joint or grip tightly. Moderate to severe weakness of C6-8 myotomes was observed, as well as the hypoesthesia over the C6-7 dermatomes. The biceps reflex was absent. The electrophysiological examination revealed decreased motor ampltitudes of axillary, musculocutaneous and median nerve $(4.4,3.6$ and $1.4 \mathrm{mV}$, respectively) and the absence of median and radial nerve SNAPs. The needle EMG revealed many positive sharp waves in the right deltoid, biceps, extensor digitorum communis, abductor pollicis brevis and C6 paraspinal muscles. These findings were consistent with a right incomplete C6-8 radiculoplexopathy. Brachial plexus MRI showed hyperintensity of right brachial plexus especially at the C6-8 nerve roots level. Consequently, clinical one-year follow-up revealed that the patient was still not able to elevate her right arm to the shoulder level. Also, she presented with numbness of the thumb and post-herpetic neuralgia.

\section{Case 7}

A 61-year old man developed burning pain and vesicular rash over left thumb, index finger and forearm. Fifteen days after his rash, he noted weakness in his left hand dorsal stretch and grip. There was moderate weakness of muscles in left C6-8 myotomes and hypoesthesia over the thumb. The triceps muscle stretch reflex was absent. The electrophysiological examination revealed decreased amplitude radial CMAPs $(4.1 \mathrm{mV})$ as compared to the contralateral side $(11.1 \mathrm{mV})$ and decreased amplitudes median and radial nerve SNAPs. The needle EMG revealed many positive sharp waves in the left extensor digitorum communis, brachioradialis, abductor pollicis

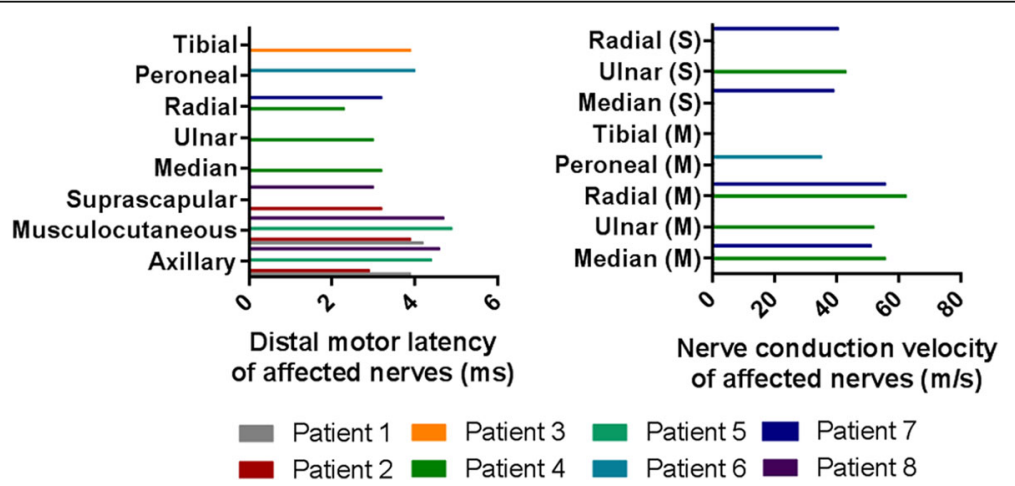

Fig. 3 Distal motor latency and nerve conduction velocity of affected nerves. The distal motor latencies were all shorter than $130 \%$ of the upper limit of normal. The nerve conduction velocities were all above $35 \mathrm{~m} / \mathrm{s}$. M: motor nerve; S: sensory nerve 

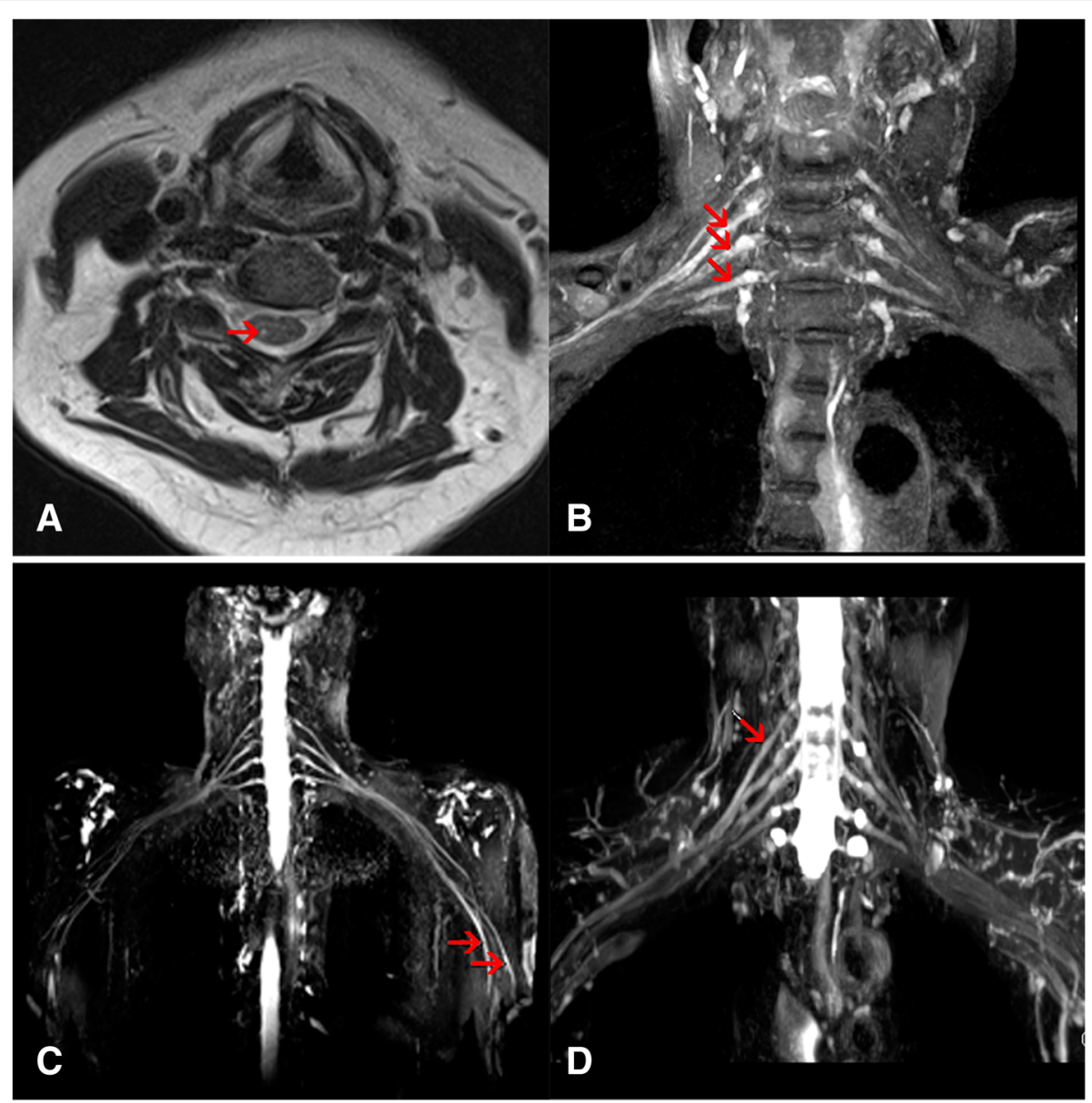

Fig. 4 Imaging characteristics of patients with SZP. Axial T2-weighted image showed the unilateral hyperintensity in the dorsal horn of C5 spinal cord in patient 2 (a). Brachial plexus magnetic resonance imaging showed hyperintensity of C6-8 nerve roots in patient 5 (b), left median and radial nerves in patient 7 (c) and C5 nerve roots in patient 8 (d)

brevis and C7 paraspinal muscles. These findings were consistent with a left median and radial nerve lesions and nerve root lesion. Moreover, brachial plexus MRI showed hyperintensity of distal median and radial nerve. Though, there was no nerve enlargement or T2 hyperintensity of nerve roots. A half-year follow-up revealed that his muscle strength did not recover, and his muscles seemed atrophied. Also, he developed post-herpetic neuralgia.

\section{Discussion}

In the present study, we confirmed that varicella-zoster virus can lead to SZP which is an uncommon complication of a common ailment, including nerve roots, brachial or lumbar plexus, and peripheral nerves lesions. The electrophysiologic and imageological findings can help demonstrate the extent and severity of affected nerves or nerve roots.

The elderly and immunocompromised patients are most susceptible to VZV $[1,9,19]$. Due to the immunosenescence in aged people, $\mathrm{HZ}$ infection frequently occurs [1]. It is a high incidence of disease over the age of 40; the highest incidence is found in the age group from 60 to 70 . [2]. The average age in our sample was 69 years old.

Yoleri et al. have suggested that zoster paresis preferentially strikes the upper limbs and then the lower limbs [8], while according to other studies, the upper and lower extremities are equally affected $[3,20]$. In the present study, upper limbs were involved in 6 out of 8 patients; which was in line with the former results. The weakness in upper limbs most commonly occurs in C57 segments, while the weakness in lower extremities most frequently occurs in L1-4 segments [5]. The involvement of C8 myotome is relatively rare [10]. In other words, the distribution of muscle weakness is common in the proximal muscles of the limbs and limb-girdles. In most of our cases, the upper extremities were involved, which was in accordance with this pattern; although in 3 patients the distal muscles were also 
involved. In 2 cases of affected lower limbs, weakness did not fit this pattern and indicated the distal weakness. Interestingly, right limbs were involved in 7 out of 8 patients, which was in accordance with previous literatures [5-18, 21-27].

Our electrophysiological study manifested low amplitudes of CMAPs or SNAPs together with many spontaneous activities in all patients, thus suggesting motor and sensory axonopathy. In axonal loss lesions, NCS and DML were normal or mildly slow. Generally, NCV was above $35 \mathrm{~m} / \mathrm{s}$ and DML was shorter than $130 \%$ of the upper normal limit in axonal loss lesions. All our results from 8 patients met the criteria. Consequently, peripheral neuropathy caused by $\mathrm{HZ}$ with predominant axonopathy could be seen through our cases electrophysiologically. Sachs et al. have reported an electrophysiological study of zoster paresis for 22 months of follow-up, which appeared to be axonopathy and reinnervation [28].

The dorsal root ganglion is the seat of VZV previous to the $\mathrm{HZ}$ eruption; therefore, it is no wonder that amplitudes of SNAPs were low, showing sensory neurons or axons damage. According to existing pathologic studies, demyelination, axon degeneration and lymphocyte infiltration can be found in affected nerves, dorsal root ganglions and dorsal horns [29]. These findings were observed in the postmortem examinations long time after $\mathrm{HZ}$ infections. Thus, it's possible that the demyelination found in autopsy is secondary to the axon degeneration induced by HZ infection. Meanwhile, our electrophysiological examinations were performed no more than 2 weeks from the onset of weakness. Axonal degeneration may be the early presentation.

Brachial or lumbosacral plexus was affected in 5 out of 8 patients, suggesting the dorsal root ganglion (the seat of virus prior to its eruption) and ventral roots lesions. Although the precise mechanism of SZP remains unclear, it is easy to understand that the spread of VZV along nerve fibers to ventral root, ventral horn, and distal nerves can lead to corresponding lesions.

Four of our patients underwent MRI examination. MRI is a useful tool for diagnosing SZP. The imaging abnormalities included nerve enlargement and T2 signal hyperintensity of dorsal horn and brachial plexus or peripheral nerves, which were consistent with clinical symptoms and elcetrophysiological findings, although these imaging manifestations are not specific and could be found in peripheral nerve inflammation caused by other diseases such as neurobrucellosis [30]. The imaging abnormalities were not found in $100 \%$ of patients, while nerve $\mathrm{T} 2$ hyperintensity or nerve enlargement was found in $70 \%$ of patients [31]. Our patent 7 did not show imaging abnormalities at roots.

In the literature, the prognosis for SZP is generally favorable, but the return of motor function can be incomplete, while recovery time can significantly vary $[10,32]$. Two-thirds of their patients had completely or almost fully recovered within a year while the other one-sixth of patients suffered from permanent weakness of extremities, which usually occurred in muscles like diaphragm, anterior tibial and the hand intrinsic muscles [2]. In our study, anterior tibial was involved in 1 patient (case 3), and hand intrinsic muscles were involved in 3 patients (case 4, 5 and 7). Nevertheless, C5-7 myotome muscles were mainly observed, even though not mentioned previously, in patient 2 who still had a poor recovery over 2.0-year follow-up. These patients had a longer recovery time than the 47 -year old man. The other reason may be probably due to the older age (patient 2-7) and diabetes mellitus (patient 2-5) [14, 33].

Our study is limited by a small sample size of this rare complication of $\mathrm{HZ}$ and retrospective design. Some patients without clinical zoster paresis were not examined with EMG, so there might be electrophysiological abnormalities in these patients. In addition, some patients were likely to have a longer follow-up for prognostic estimation.

\section{Conclusions}

In summary, the present study highlights clinical, electrophysiological and imaging characteristics of this rare motor involvements of HZ. It is associated with significant limb weakness, obvious nerve axons lesion, and it is localized in nerve roots, plexus or peripheral nerves. The information is crucial for neurologists in order to avoid unnecessary misdiagnosis. Also, SZP should be considered in the differential diagnosis of acute painful motor weakness of limbs. The electrophysiological testing and MRI scan can be useful for confirming the diagnosis of SZP.

\section{Abbreviations \\ CMAPs: Compound muscle action potentials; DML: Distal motor latency; EMG: Electromyography; HZ: Herpes zoster; MRI: Magnetic resonance imaging; MUAPs: Motor unit action potentials; NCS: Nerve conduction studies; NCV: Nerve conduction velocity; SNAPs: Sensory nerve action potentials; SZP: Segmental zoster paresis; VZV: Varicella-zoster virus}

\section{Acknowledgments \\ The authors thank Chun-Lin Yang and Min Zhang at Department of Neurology, Shandong Provincial Qianfoshan Hospital, Shandong University, for assistance with the preparation of this manuscript. \\ Funding \\ This work was partially supported by Taishan Scholars Construction Engineering of Shandong Province (NO. ts20130914), Medicine and Health Science Technology Development Programme of Shandong Province (NO. 2015WS0226) and Key Research and Development Plan of Shandong Province (NO. GG201709190225).}

\section{Availability of data and materials}

The datasets used and analyzed during the current study are available from the corresponding author on reasonable request. 


\section{Authors' contributions}

$Y L$ and RSD participated in the conception and the design of the study. YL drafted the manuscript. BYW, JJX, BY and HL participated in the acquisition of raw data and the analysis of the clinical data. ZSM performed and interpreted the imaging data. RSD provided the major funding for the study. All authors read and approved the final manuscript.

\section{Ethics approval and consent to participate}

This study was approved by the ethic committee of the Shandong Provincial Qianfoshan Hospital, Shandong University. Six patients signed the informed consents and two patients were not contacted before the submission. Exception to the requirement of informed consent was obtained from the ethic committee due to the retrospective study.

\section{Consent for publication}

Written informed consent was obtained from the patients described in cases (case 1, 2, 3, 5, 7and 8) for the publication of their personal and medical information and any accompanying images. A copy of the written consent is available for review by the Editor of this journal.

\section{Competing interests}

The authors declare that they have no competing interests.

\section{Publisher's Note}

Springer Nature remains neutral with regard to jurisdictional claims in published maps and institutional affiliations.

\section{Author details}

'Department of Electromyography, Shandong Provincial Qianfoshan Hospital, Shandong University, Jinan 250014, People's Republic of China. ${ }^{2}$ Department of radiology, Shandong Provincial Qianfoshan Hospital, Shandong University, Jinan 250014, People's Republic of China. ${ }^{3}$ Department of Neurology, Shandong Provincial Qianfoshan Hospital, Shandong University, Jinan 250014, People's Republic of China.

Received: 23 April 2018 Accepted: 15 August 2018

Published online: 21 August 2018

\section{References}

1. Yawn BP, Gilden D. The global epidemiology of herpes zoster. Neurology. 2013;81(10):928-30.

2. Gupta SK, Helal BH, Kiely P. The prognosis in zoster paralysis. J Bone Joint Surg Br. 1969;51(4):593-603.

3. Thomas JE, Howard FM Jr. Segmental zoster paresis--a disease profile. Neurology. 1972;22(5):459-66.

4. Jones LK Jr, Reda H, Watson JC. Clinical, electrophysiologic, and imaging features of zoster-associated limb paresis. Muscle Nerve. 2014;50(2):177-85.

5. Kawajiri S, Tani M, Noda K, Fujishima K, Hattori N, Okuma Y. Segmenta zoster paresis of limbs: report of three cases and review of literature. Neurologist. 2007:13(5):313-7.

6. Reda H, Watson JC, Jones LK Jr. Zoster-associated mononeuropathies (ZAMs): a retrospective series. Muscle Nerve. 2012:45(5):734-9.

7. Kim JG, Chung SG. Herpetic brachial plexopathy: application of brachial plexus magnetic resonance imaging and ultrasound-guided corticosteroid injection. Am J Phys Med Rehabil. 2016;95(5):e67-71.

8. Yoleri O, Olmez N, Oztura I, Sengul I, Gunaydin R, Memis A. Segmental zoster paresis of the upper extremity: a case report. Arch Phys Med Rehabil. 2005;86(7):1492-4.

9. Rastegar S, Mahdavi SB, Mahmoudi F, Basiri K. Herpes zoster segmental paresis in an immunocompromised breast cancer woman. Adv Biomed Res. 2015;4:170.

10. Kreps $C E$, Rynders SD, Chhabra AB, Jenkins JG. C8 myotome herpes zoster paresis. Am J Orthop. 2012;41(5):220-2.

11. Teo HK, Chawla M, Kaushik M. A rare complication of herpes zoster: segmental zoster paresis. Case Rep Med. 2016;2016:7827140.

12. Park SE, Ganji P, Ji JH, Park SH. Transient motor paresis caused by herpes zoster. J Shoulder Elb Surg. 2016;25(10):e309-12.

13. Martic V. Recurrent herpes zoster with segmental paresis and postherpetic neuralgia. Vojnosanit Pregl. 2014;71(2):214-7.

14. Namekawa M, Kameda T, Kumabe A, Mise J. Segmental zoster paresis of the right shoulder. Intern Med. 2013;52(24):2839.
15. Kang SH, Song HK, Jang Y. Zoster-associated segmental paresis in a patient with cervical spinal stenosis. J Int Med Res. 2013:41(3):907-13.

16. Yoshioka M, Kurita Y, Hashimoto M, Murakami M, Suzuki M. A case of segmental zoster paresis with enhanced anterior and posterior spinal roots on MRI. J Neurol. 2012;259(3):574-5.

17. Khan A, Camilleri J. Motor radiculopathy. BMJ Case Rep. 2012;2012:bcr2012006246.

18. Umehara T, Sengoku R, Mitsumura H, Mochio S. Neurological picture. Findings of segmental zoster paresis on MRI. J Neurol Neurosurg Psychiatry. 2011;82(6):694

19. Andrei G, Snoeck R. Advances in the treatment of varicella-zoster virus infections. Adv Pharmacol. 2013:67:107-68.

20. Cruz-Velarde JA, Munoz-Blanco JL, Traba A, Nevado C, Ezpeleta D. Segmental motor paralysis caused by the varicella zoster virus. Clinical study and functional prognosis. Rev Neurol. 2001;32(1):15-8.

21. Chabot RH, Wirtz PW. Teaching Neurolmages: MRI findings in varicella zoster brachial plexus neuritis. Neurology. 2011;76(15):e76.

22. Alshekhlee A, Tay E, Buczek M, Shakir ZA, Katirji B. Herpes zoster with motor involvement: discordance between the distribution of skin rash and localization of peripheral nervous system dysfunction. J Clin Neuromuscul Dis. 2011:12(3):153-7.

23. Samuraki M, Yoshita M, Yamada M. MRI of segmental zoster paresis. Neurology. 2005:64(7):1138.

24. Yaszay B, Jablecki CK, Safran MR. Zoster paresis of the shoulder. Case report and review of the literature. Clin Orthop Relat Res. 2000;377:112-8.

25. Hanakawa T, Hashimoto S, Kawamura J, Nakamura M, Suenaga T, Matsuo M. Magnetic resonance imaging in a patient with segmental zoster paresis. Neurology. 1997;49(2):631-2.

26. Fabian VA, Wood B, Crowley P, Kakulas BA. Herpes zoster brachial plexus neuritis. Clin Neuropathol. 1997;16(2):61-4.

27. Braverman DL, Ku A, Nagler W. Herpes zoster polyradiculopathy. Arch Phys Med Rehabil. 1997:78(8):880-2.

28. Sachs GM. Segmental zoster paresis: an electrophysiological study. Muscle Nerve. 1996:19(6):784-6.

29. Watson CP, Deck JH, Morshead C, Van der Kooy D, Evans RJ. Post-herpetic neuralgia: further post-mortem studies of cases with and without pain. Pain. 1991;44(2):105-17.

30. Al-Sous MW, Bohlega SA, Al-Kawi MZ, McLean DR, Ghaus SN Polyradiculopathy. A rare complication of neurobrucellosis. Neurosciences (Riyadh). 2003:8(1):46-9.

31. Zubair AS, Hunt C, Watson J, Nelson A, Jones LK Jr. Imaging findings in patients with zoster-associated plexopathy. AJNR Am J Neuroradiol. 2017; 38(6):1248-51.

32. Merchut MP, Gruener G. Segmental zoster paresis of limbs. Electromyogr Clin Neurophysiol. 1996:36(6):369-75.

33. Mondelli M, Romano C, Rossi S, Cioni R. Herpes zoster of the head and limbs: electroneuromyographic and clinical findings in 158 consecutive cases. Arch Phys Med Rehabil. 2002;83(9):1215-21.

\section{Ready to submit your research? Choose BMC and benefit from:}

- fast, convenient online submission

- thorough peer review by experienced researchers in your field

- rapid publication on acceptance

- support for research data, including large and complex data types

- gold Open Access which fosters wider collaboration and increased citations

- maximum visibility for your research: over $100 \mathrm{M}$ website views per year

At BMC, research is always in progress.

Learn more biomedcentral.com/submissions 